\title{
Effets de la température, du pH et du rayonnement solaire sur la survie de différentes bactéries d'intérêt sanitaire dans une eau usée épurée par lagunage Effets de la température, du pH et du rayonnement solaire sur la survie de différentes bactéries d'intérêt sanitaire dans une eau usée épurée par lagunage
}

\author{
N. Mezrioui et B. Baleux
}

Volume 5, numéro 4, 1992

URI : https://id.erudit.org/iderudit/705148ar

DOI : https://doi.org/10.7202/705148ar

Aller au sommaire du numéro

\section{Éditeur(s)}

Université du Québec - INRS-Eau, Terre et Environnement (INRS-ETE)

ISSN

0992-7158 (imprimé)

1718-8598 (numérique)

Découvrir la revue

Citer cet article

Mezrioui, N. \& Baleux, B. (1992). Effets de la température, du pH et du rayonnement solaire sur la survie de différentes bactéries d'intérêt sanitaire dans une eau usée épurée par lagunage. Revue des sciences de l'eau / Journal of Water Science, 5(4), 573-591. https://doi.org/10.7202/705148ar
Résumé de l'article

L'effet du pH $(6.2,7.2,8.4,9.6)$, de la température $\left(4^{\circ} \mathrm{C}, 12^{\circ} \mathrm{C}, 23^{\circ} \mathrm{C}\right)$ et du rayonnement solaire a été étudié expérimentalement sur les évolutions des abondances d'Escherichia coli 0126 : B16 et d'autres bactéries pathogènes d'intérêt sanitaire : Salmonella typhimurium et Aeromonas hydrophila. En eau usée épurée, les résultats obtenus montrent que la température et le rayonnement solaire sont parmi les facteurs responsables des variations des abondances de ces bactéries dans les milieux aquatiques. Les faibles valeurs de température $\left(4^{\circ} \mathrm{C}\right)$ favorisent la survie d'E. coli et de S. lyphimurlum et réduisent celle d'A. hydrophila. Les faibles valeurs de température $\left(4^{\circ} \mathrm{C}\right)$ augmentent non seulement la survie bactérienne d'E. coli et de S. typhimurium mais limitent les effets nocifs des $\mathrm{pH}$ alcalins ( $\mathrm{pH}$ 9.6) sur la diminution des abondances de ces bactéries. Parmi les pH alcalins étudiés, le pH 9.6 entraîne la plus forte diminution du temps de survie vis-à-vis d'E. coli, de A. hydrophila et de S. typhimarlam. A pH 9.6, les $\mathrm{T}_{90}$ obtenus à une température de $12^{\circ} \mathrm{C}$ sont respectivement de 23,20 et 33 heures. Le rayonnement solaire joue également un rôle important dans la réduction des abondances bactériennes et ce d'autant plus que le pH est élevé. Dans l'eau usée épurée par lagunage, ajustée à $\mathrm{pH} 9.6$ et exposée au rayonnement solaire, les $\mathrm{T}_{90} \mathrm{~d}^{\prime} E$ coli, d'A. hydrophila et de S. typhimarium sont respectivement de 6, 4 et de 6 heures. L'effet combiné $\mathrm{du} \mathrm{pH}$ et du rayonnement est beaucoup plus important sur la réduction des abondances bactériennes que si l'un des facteurs agit isolément.
Tous droits réservés @ Revue des sciences de l'eau, 1992
Ce document est protégé par la loi sur le droit d'auteur. L’utilisation des services d’Érudit (y compris la reproduction) est assujettie à sa politique d'utilisation que vous pouvez consulter en ligne.

https://apropos.erudit.org/fr/usagers/politique-dutilisation/ 


\title{
Effets de la température, du $\mathrm{pH}$ et du rayonnement solaire sur la survie de différentes bactéries d'intérêt sanitaire dans une eau usée épurée par lagunage
}

\author{
Effects of temperature, $\mathrm{pH}$ and solar radiation on survival \\ of sanitary interest bacteria, in waste water treated \\ by purifying lagoon
}

N. MEZRIOUI', B. BALEUX²

Reçu le 6 décembre 1991, accepté pour publication le 3 juillet 1992*.

SUMMaAY

The behaviour of coliforms especially $E$. coli, pathogenic bacteria (Salmonella typhimurium), and occasionally pathogenic bacteria (Aeromonas hydrophila) in different aquatic environments has often been studied, but rather from a medical than an environmental point of view. Hawever, studies on the effect of various environmental factors such as temperature, $\mathrm{pH}$ and solar radiation on their evolution have seldom been carried out. Their action on pathogenic and occasional pathogenic bacteria remains fragmentary.

The aim of this survey is analyse the effect (in laboratory experiment) of certain environmental factors such as temperature, $\mathrm{pH}$ and solar radiation on the behaviour of $E$. colf 0126:B16, S. typhimurium and $A$. hydrophila, in waste waters treated in a sewage treatment lagoon. The values of these tested factors are comparable to those which exist in aquatic environments at different seasons.

Experimentation has been realized in a variable and complex medium in this case water from the basin of lagoon, in order to study the varying effects of differents factors on evolution of ahundance bacteria. Hierarchization of effects of environmental factors or of certain of their associations has been proposed.

In order to study the action of the temperature and the combined action of temperature-ph on the bacterial evolution, four sterile 1 litre flasks were filled with $500 \mathrm{ml}$ of waste waler treated in lagoon. The $\mathrm{pH}$ of each flask was adjus ted by acid $\left(\mathrm{H}_{2} \mathrm{SO}_{4}\right)$ or alkaline ( $\left.\mathrm{KaOH}\right)$ solutions at $6.2 ; 7.2 ; 8.4$ and 9.6 . Each flask was seeded with a bacterial inoculum of each of the tested strains

1. Laboratoire de Microbiologie, Département de biologie, Université CADI AYYAD, Faculté des Sciences, Marrakech,Maroc.

2. Laboratoire d'Hydrobiologie URACNRS 1355, Université des Sciences et Techniques du Languedoc, 34095 , Montpellier Cedex, France.

* Les commentaires seront regus jusqu'au 15 juin 1993. 
(E. coli, A. hydrophila and S. typhimurium). The temperature used for the incu bation of the flasks corresponded to the different seasons : $4^{\circ} \mathrm{C}, 12^{\circ} \mathrm{C}$ and $23{ }^{\circ} \mathrm{C}$. The temporal evolution of the abundance of each tested strain is followed up by the indirect counting on specific media : TC and Tergitol Lactose Agar (Institut Pasteur Production) and incubation at $44.5^{\circ} \mathrm{C}$ for 24 hours ( $E$. coli), Salmonella-Shigella Agar (Biokérieux) and incubation at $37^{\circ} \mathrm{C}$ for 24 hours (S. typhimurium 4,5 H 1,2 i), Pril-Xylose-Ampicilin agar (ROGOL et al., 1979) and incubation at $37^{\circ} \mathrm{C}$ for 48 hours (A. hydrophila ATCC 7966).

The action of the solar radiation and the combined action of solar radiation-pH on E. coli, S. typhimurium and A. hydrophila was carried out as follows:

i) 3 series of $\mathbf{2}$ glass crystallizers were filled with $500 \mathrm{ml}$ of waste water trea ted in lagoon (height water: $55 \mathrm{~mm}$ ). Three reactional mediums were thus prepared by adjusting the pH to $7.2 ; 8.4$ and 9.6 . Each $\mathrm{pH}$ corresponds to two crystailizers which were seeded with a bacterial inoculum of $E$. colf; $S$. typhimurium and A. hydrophila. For each pH one of the crystallizers is placed in front of a window receiving plenty of solar radiation, the other being protected from all radiation by aluminium paper and used as control. The number of bacteria is verified by counting the colony forming units (c.f.u.) through a dilutionspreading technique on the same media as those used to estimate the action of temperature and pH on the survival of E. coli, A. hydrophils and S. typhimurium.

ii) In order to understand the night and day variations in the number of $E$. coli according to the solar radiation, another method was used. A standard $E$. coli inoculum is added to some water of the outflow of lagoon filtered on $0,45 \mu \mathrm{m}$ (Millipore) and exposed to solar radiation. The temporal evolution of the number of $E$. coli is then followed by daily counts at 6:00 AM, at 2:00 PM and at 19:00 PM (GMT hour).

Comparison of the different results is made with $T_{90}$ (the time needed to reduce the initial bacterial population by $90 \%$ ) and with an equality test of 2 regression coefficients (FRONTER, 1981).

Obtained results show that temperature and solar radiation could be considered among factors responsible for the variable abundance of $E$. coli, A. hydrophila and $S$. typhimurium. At pH $6.2 ; 7.2$ and 8.4 and after incubation at $23^{\circ} \mathrm{C}$, the survival time $\left(T_{90}\right)$ of $E$. coli and $A$. hydrophila is almost the same $\left(T_{90}=53 \mathrm{~h}\right)$ and is anyway inferior to the one for $S$. typhimurium which survi ved nearly twice as long $\left(T_{m}=100 \mathrm{~h}\right)$. On the other hand, at pH 9.6 and with the same incubation temperature of $23^{\circ} \mathrm{C}$, the survival time of different tested bacteria is reduced. The $T_{n 0}$ for $E$. coli is the same as the one for $A$. hydrophila $\left(T_{90}=20 \mathrm{~h}\right.$ ) and is 24 hours for $S$. typhimurion (table 1$)$. It is almost 4,3 times lower than the one with pH 6.2 and 7.2 .

The evolutions in number of the same hacteria with similar $\mathrm{pH}$ but at a temperature of $12^{\circ} \mathrm{C}$ are shown in figure 2 . Compared with results obtained at $23^{\circ} \mathrm{C}$, one notices that the survival of different tested bacteria is longer. The survival times for $E$. coli and $A$. hydrophila are almost the same $\left(T_{e 0}=73 \mathrm{~h}\right)$, but differ from the one for $S$. byphimurium $\left(T_{90}=142 \mathrm{~h}\right)$, a bacteria which always survives better than other bacteria under the same experimental conditions. Low temperatures $\left(4^{\circ} \mathrm{C}\right)$ increase the survival of $E$. coli and Salmonella typhimurium and reduce that of Aeromonas hydrophila. Not only do these low temperatures $\left(4^{\circ} \mathrm{C}\right)$ reduce survival of $E$. coli and $S$. typhimurium but they also reduce negative effects of alkaline $\mathrm{pH}$ (pH 9.6) which decreases the abundance of these bacteria. Among the alkaline pH values studied, pH 9.6 caused the highest reduction of the survival of $E$. coli, A. hydrophila and S. typhimurium.

We can conclude that at $4^{\circ} \mathrm{C}$ the survival time of $A$. hydrophila significantly decreases in comparison with that of $E$. coli and $S$. typhimurium. At 12 and $23^{\circ} \mathrm{C}$, the survival time of $E$. coli and A. hydrophila is the same. S. typhimurium 
Survived better than both $\boldsymbol{E}$. coli and $\boldsymbol{A}$. hydrophila. Solar radiation is also considered an important factor in the decrease of bacterial abundance especially when the $\mathrm{pH}$ is alkaline. Under solar radiation and $\mathrm{pH} \mathrm{9.6,} \mathrm{the}$ survival time of $E$. coli, $A$. hydrophila and $S$. typhimurium is respectively 6,4 and 6 hours (table 2). The decline in the numbers of these bacteria in effluent lagoon samples was found to be significantly greater in the presence of both pH-temperature or pH-solar radiation than when each of these factors was acting independently.

In waste waters treated in lagoon and under sunlight, $E$. coli 0126 : B16 undergoes a successive evolution (night growth phase-diurnal decrease phase: fig. 5). According to all coumt values, we can plot the regression straight line ( $\log 10$ bacteria $=$ rs (time)) and estimate the death coefficient which is the slope of this regressional straight line. This model expresses the tendency of the linear decrease of $E$. coli. Predictions of this model are satisfactory for counting values corresponding to periods of 24 hours or their multiple. The decrease phase or stable night phase cannot be predicte by this model owing to the fact that it is linear and presents the negative slope.

Key words : lagoon system, temperature, $\mathrm{pH}$, sunlight, survival, E. coli, pathogenic bacteria.

RESUME

L'effet du pH $(6.2,7.2,8.4,9.6)$, de la température $\left(4^{\circ} \mathrm{C}, 12^{\circ} \mathrm{C}, 23^{\circ} \mathrm{C}\right)$ et du rayonnement solaire a été étudié expérimentalement sur les évolutions des abondances d'Escherichia coli 0126: B16 et d'autres bactéries pathogènes d'intérêt sanitaire : Salmonella typhimurium et Aeromonas hydrophila. En eau usée épurée, les résultats obtenus montrent que la température et le rayonnement solaire sont parmi les tacteurs responsables des variations des abondances de ces bactéries dans les milieux aquatiques. Les faibles valeurs de température $\left(4^{\circ} \mathrm{C}\right)$ favorisent la survie d'E. coli et de $S$. typhimurium et rédui sent celle d'A. hydrophila. Les faibles valeurs de température $\left(4^{\circ} \mathrm{C}\right)$ augimentent non seulement la survie bactérienne d'E. coli et de $S$. byphimurium mais limitent les effets nocifs des $\mathrm{pH}$ alcalins (pH 9.6) sur la diminution des abondances de ces bactéries. Parmi les pH alcalins étudiés, le pH 9.6 entraîne la plus forte diminution du temps de survie vis-d̀-vis d'E. colf, de A. hydrophila et de $S$. typhimurium. A pH 9.6, les $\mathrm{T}_{90}$ obtenus à une température de $12{ }^{\circ} \mathrm{C}$ sont respectivement de 23, 20 et 33 heures. Le rayonnement solaire joue également un rôle important dans la réduction des abondances bactériennes et ce d'autant plus que le pH est ćlevé. Dans l'eau usée épurée par lagunage, ajustée a ph 9.6 et exposée au rayonnement solaire, les $T_{n g}$ d'E. colf, d'A. hydrophila et de $S$. typhimurium sont respectivement de 6,4 et de 6 heures. L'effet combiné du pH et du rayonnement est beaucoup plus important sur la réduction des abondances bactériennes que si l'un des facteurs agit isolément.

Mots clés : lagunage, temperature, pH, rayonnement solaire, survie, E. coli, pathogènes.

\section{1 - INTRODUCTION}

Le comportement des bactéries. témoins de contamination fécale, des bactéries pathogènes (Salmonella typhimurium) et pathogènes opportunistes (Aeromonas hydrophila) dans les différents milieux aquatiques, a fait déjà 
l'objet d'études mais davantage dans une approche sanitaire qu'environnementale (MITCHEL et STARZYK, 1975). Cependant les études de leur survie en relation avec les effets de différents facteurs environnementaux tels que la température, le $\mathrm{pH}$ et le rayonnement solaire sont peu nombreuses. La température est l'un des paramètres faisant partie des modèles explicatifs des évolutions des abondances bactériennes (DAVEY, 1989). L'effet de la température sur $E$. coli a été étudié expérimentalement par MC FETERS et STUART (1972) dans l'eau douce d'une rivière. Ces auteurs ont noté une relation inverse entre l'abondance de cette bactérie et l'augmentation de la température, surtout dans la gamme comprise entre 5 et $30^{\circ} \mathrm{C}$. La survie d'E. coli dans l'eau de mer est d'autant plus longue que la température est faible (VASCONCELOS et SWARTZ, 1976). En ce qui concerne l'effet de la température sur la survie bactérienne en milieu marin, GRAHAM et SIEBURT (1973) ont remarqué qu'une augmentation de la température de 15 à $25^{\circ} \mathrm{C}$, sans ajout de substances nutritives, permet une décroissance de $S$. typhimurium et d'E. coli. Quant à $A$. hydrophila ROUF et RIGNEY (1971) ont montré qu'en milieu de culture liquide cette bactérie peut se développer dans un large éventail de température $\left(\mathrm{O}\right.$ à $55^{\circ} \mathrm{C}$ ) avec un optimum situé entre 15 et $20^{\circ} \mathrm{C}$.

L'action du rayonnement solaire sur la disparition bactérienne a été décrite depuis longtemps. Les effets du rayonnement solaire sur les coliformes fécaux notamment $E$. coli ont fait l'objet de nombreuses études (CHAMBERLIN et MIT CHELL, 1978 ; FUJIOKA et al., 1981 et GAMESON et GOULD, 1985). MARTIN et al. (1985) ont montré l'importance du rayonnement solaire dans la disparition des coliformes dans l'eau de mer sous les effets non seulement des UV mais aussi des rayonnements en lumière visible. Selon MOELLER et CALKINS (1980), TROUSSELLIER et al. (1986), le rayonnement solaire joue également un rôle important dans la disparition des bactéries de contamination fécale au cours de l'épuration des eaux usées par lagunage. Toutefois les études portant sur l'action du rayonnement solaire sur les bactéries pathogènes ou pathogènes opportunistes restent fragmentaires.

Le but de cette étude expérimentale est d'analyser les effets de certains facteurs environnementaux tels que la température, le $\mathrm{pH}$ et le rayonnement solaire, sur le comportement d'une bactérie témoin de contamination fécale : Escherichia coli, d'une bactérie pathogène: Salmonella typhimurium et d'une bactérie pathogène opportuniste: Aeromonas hydrophila dans des eaux usées épurées par un système de traitement par lagunage. Les valeurs de ces différents facteurs testés sont comparables à celles qui existent dans les milieux aquatiques aux différentes saisons.

L'expérimentation a été réalisée en milieu complexe et variable en l'occurence une eau de bassins de lagunage et de ce fait, seuls ont été pris en compte les effets des variations des différents facteurs environnementaux sur les évolutions des abondances des variables bactériennes. Une hiérarchisation des effets des facteurs environnementaux ou de certaines de leur associations est proposée. 


\section{2 - MATÉRIEL ET MÉTHODES}

Le milieu réactionnel choisi est l'eau de l'effluent d'un lagunage, ouvrage épurateur des eaux usées de la commune de Mèze : latitude $43^{\circ}$ nord et $3^{\circ}$ sud : France (BALEUX et TROUSSELLIER, 1983 ; LEGENDRE et al., 1984).

\section{Souches bactériennes testées}

Les souches étudiées sont $\dot{E}_{\text {. }}$ coli O126:B16 (témoin de contamination fécale), S. typhimurium 4,5 H1, 2i (pathogène) et $A$. hydrophila ATCC 7966 (pathogène opportuniste). Pour chaque souche testée, une culture bactérienne confluente sur milieu gélosé (Gélose Nutritive : BioMérieux) préalablement incubée pendant 18 heures à $37^{\circ} \mathrm{C}$, est mise en suspension dans de l'eau physiologique $(9 \% \mathrm{Nacl})$. A partir de cette suspension mère, différentes dilutions sont effectuées dans de l'eau physiologique pour avoir un nombre suffisant de cellules avec lesquelles sont ensemencés les différents milieux réactionnels étudiés.

\section{Action du pH et de la température}

Trois séries de 4 flacons (1 flacon par pH étudié) de capacité de 1 litre en verre borosilicaté contenant chacun $500 \mathrm{ml}$ d'un même prélèvement d'eau usée épurée par lagunage et non stérilisée (eau telle quelle) sont ajustés à l'aide de solutions acide $\left(\mathrm{H}_{2} \mathrm{SO}_{4}\right)$ ou alcaline $(\mathrm{NaOH})$, à pH $6.2 ; 7.2 ; 8.4$ et 9.6. Chacune des séries est ensemencée à la fois avec une suspension d'E. coli O126: B16 (concentration finale dans chaque flacon est équivalente à $1.10^{4}$ u.f.c. $\mathrm{ml}^{-1}$ ) avec $S$. typhimurium (concentration finale/flacon : 4,6.103 u.f.c. $\mathrm{ml}^{-1}$ ) et avec $A$. hydrophila (concentration finale/flacon : $2.10^{3}$ u.f.c. $\mathrm{ml}^{-1}$ ). Chacune des souches bactériennes ensemencées dans les 4 flacons aux différents pH sont incubées, à l'obscurité, à 3 températures différentes : $4^{\circ} \mathrm{C}$, $12^{\circ} \mathrm{C}$ et $23^{\circ} \mathrm{C}$ correspondant aux différentes températures moyennes saisonnières (hiver, printemps et automne, été) relevées dans les bassins de lagunage de Mèze. Les évolutions temporelles des abondances des souches bactériennes sont suivies avec un pas de temps de 24 heures pendant 7 jours par dénombrement indirect sur milieux de culture spécifiques par les techniques de dilutions-étalement et/ou filtration sur membrane sur milieux : gélose lactosée au TTC et Tergitol (Institut Pasteur Production) incubée à $44,5^{\circ} \mathrm{C}$ pendant 24 heures $(E$. coli), gélose Salmonella-Shigella agar (bioMerieux) incubée à $37^{\circ} \mathrm{C}$ pendant 24 heures (S. typhimurium), Pril-Xylose-Ampicilline agar (ROGOL et al. 1979), incubée à $37^{\circ} \mathrm{C}$ pendant 48 heures (A. hydrophila).

\section{Action du pH et du rayonnement solaire}

Pour évaluer l'effet du rayonnement solaire ou l'effet combiné du rayonnement-pH sur la survie bactérienne, deux expériences ont été réalisées :

i) 3 séries de 2 cristallisoirs de verre de diamètre de $120 \mathrm{~mm}$ remplis chacun avec un volume de $500 \mathrm{ml}$ (hauteur d'eau : $55 \mathrm{~mm}$ ) d'un même prélèvement d'eau usée épurée par lagunage et non stérilisée, sont ajustés à pH $7.2 ; 8.4$ et 9.6. A chacun des $\mathrm{pH}$ deux cristallisoirs sont ensemencés avec 
une suspension d'E. coli O126: B16 (concentration finale/cristallisoir : $3.10^{3}$ u.f.c. $\mathrm{ml}^{-1}$ ), avec une suspension de $S$. typhimurium (concentration finale/cristallisoir : $2,3.10^{3}$ u.f.c. $\mathrm{ml}^{-1}$ ), et avec une suspension d'A. hydrophila (concentration finale/cristallisoir : $4,5.10^{3}$ u.f.c. $\mathrm{ml}^{-1}$ ). Les deux cristallisoirs sont placés devant une fenêtre bien exposée aux radiations solaires (saison estivale). L'un est exposé au rayonnement solaire (jour-nuit), l'autre (témoin) est protégé de ce rayonnement par recouvrement total par du papier d'aluminium (Alupack colgate). Les évolutions des abondances bactériennes sont suivies avec un pas de temps de 24 heures pendant 5 jours par dénombrement des unités formanteolonies par les mêmes techniques et sur les mêmes milieux de cultures sélectifs que ceux utilisés pour évaluer l'action de la température et du $\mathrm{pH}$. Pendant cette période d'incubation (5 jours) à la température ambiante $\left(20-23^{\circ} \mathrm{C}\right)$, les variations du $\mathrm{pH}$ des milieux réactionnels restent faibles et ne dépassent pas 0,5 unité.

ii) En vue de vérifier si l'action du rayonnement solaire sur les abondances bactériennes variait ou non en fonction des cycles diurnes et nocturnes, un suivi journalier de ces abondances a été réalisé sur une souche d'E. coli en période estivale. Pour ce faire, deux cristallisoirs de verre de $120 \mathrm{~mm}$ de diamètre sont remplis avec $500 \mathrm{ml}$ d'eau usée épurée par lagunage $(\mathrm{pH} \mathrm{8.4)} \mathrm{et}$ stérilisée par filtration sur membrane $(0,45 \mu \mathrm{m}$ Millipore) pour éliminer l'effet des autres paramètres tels que la compétition bactérienne et la prédation. Chacun est ensemencé avec une suspension d'E. coli (concentration finale/cristallisoir : $1.10^{4}$ u.f.c. $\mathrm{ml}^{-1}$ ). L'un est exposé au rayonnement solaire en le plaçant devant une fenêtre bien exposée aux radiations solaires, l'autre est protégé des effets de ce rayonnement par un écran (papier aluminium). Les températures de l'eau relevées au cours de cette période d'étude étaient en moyenne de $18^{\circ} \mathrm{C}$ le matin et de $25^{\circ} \mathrm{C}$ l'après midi. Les évolutions des abondances bactériennes sont suivies quotidiennement à $6 \mathrm{~h}$, à $14 \mathrm{~h}$ et à $19 \mathrm{~h}$ (heures GMT) dans les deux cristallisoirs pendant 72 heures, par dénombrement bactérien (u.f.c.) sur milieu de culture sélectif (gélose Lactosée au TTC et Tergitol).

Les souches bactériennes éprouvées dans cette étude expérimentale sont des souches de collections de laboratoire ou isolées de tubes digestifs. Leur évolution d'abondance et leur survie sont-elles identiques, dans les milieux aquatiques tels que des bassins de stabilisation de lagunage, à celles de souches « sauvages " déjà adaptées à un milieu environnemental aquatique ? Pour répondre à cette question 4 cristallisoirs de verre de $120 \mathrm{~mm}$ de diamètre remplis chacun par $500 \mathrm{ml}$ d'eau usée épurée par lagunage et stérilisée par filtration (porosité $0,45 \mu \mathrm{m}$ Millipore) sont ensemencés. Deux le sont avec une souche d'E. coli O126: B16 (concentration finale/cristallisoir: $1.10^{4}$ u.f.c. $\mathrm{ml}^{-1}$ ) les deux autres avec une souche d'E. coli sauvage (concentration finale/cristallisoir : $4,210^{3}$ u.f.c. $\mathrm{ml}^{-1}$ ). Cette souche sauvage a été isolée des eaux du lagunage de Mèze à partir des colonies décrites comme coliformes fécaux sur milieu sélectif (gélose TTC et Tergitol incubation $44,5^{\circ} \mathrm{C}$ ) et confirmées comme $E$. coli (urée : - ; indole : + ; gaz : + ; VP : -).

Deux cristallisoirs sont exposés au rayonnement solaire (quantité moyenne $=495$ Joules $\mathrm{cm}^{-2}$ Jour-1), deux autres sont protégés du rayonne ment par un écran (papier aluminium) et sont dénommés : non exposés. 
Durant cette étude expérimentale, la quantité de rayonnement solaire reçu par $\mathrm{cm}^{2}$ de surface de l'échantillon exposé est mesurée par un pyranomètre type CM5700596 incorporé à un millivoltmètre.

\section{Test de comparaison des résultats}

La comparaison des différents résultats obtenus au cours de toutes ces ex. périences est faite en faisant appel au $\mathrm{T}_{90}$ (temps nécessaire à la disparition de $90 \%$ des effectifs de la population bactérienne initiale).

En assimilant la décroissance des bactéries à une cinétique du premier ordre on peut, à partir des points expérimentaux, déterminer une droite de régression : $\log$ (bactéries) $=f$ (temps), pour chaque expérience. Ainsi, on peut déterminer une pente représentant un coefficient d'abattement horaire (et aussi un $T_{90}$ ) pour chaque souche étudiée (BONNEFONT et al., 1990).

Le test d'égalité de 2 coefficients de régression (FRONTIER, 1981) tenant compte des pentes des droites de régression $(X=\log$ Nombre de bactéries et $Y=$ temps d'incubation) et des ordonnés à l'origine, permet de vérifier si deux droites comparées entre elles sont significativement différentes ou non à un seuil de probabilité donné.

\section{3 - RÉSULtaTS}

\section{pH - température}

Les évolutions temporelles des abondances d'E. coli, d'A. hydrophila ot de S. typhimurium dans l'eau usée épurée par lagunage telle quelle et incubée à la température de $23^{\circ} \mathrm{C}$ et aux $\mathrm{pH} 6.2,7.2,8.4$ et 9.6 sont variables (fig. 1). Aux pH 6.2, 7.2 et 8.4 les temps de survie d'E. coll et d'A. hydrophila sont peu différents $\left(T_{90}=53\right.$, heures, tableau 1$)$ et sont toutefois inférieurs à celui de S. typhimurium qui survit presque deux fois plus $\left(T_{90}=100 \mathrm{~h}\right)$. Par contre à $\mathrm{pH} 9.6$ et sous la même température d'incubation $\left(23^{\circ} \mathrm{C}\right)$ la survie des différentes bactéries testées se trouve réduite. Le $T_{90}$ d'E. coli et d'A. hydrophila est de 20 heures, celui de $S$. typhimurium est de 24 heures. II est donc 4,3 fois inférieur à celui obtenu aux pH 6.2, 7.2 ou 8.4. Cette meilleure survie de $S$. typhimurium comparée à celle d'E. coli a été notée par HICKEY et HIRSHFIELD, 1990 qui considèrent que Salmonella typhimurium s'adapte mieux à pH 5 que $E$. coli.

Les évolutions des abondances des mêmes bactéries aux mêmes pH mais à une température de $12^{\circ} \mathrm{C}$ sont illustrées sur la figure 2. Par comparaison avec les résultats obtenus à $23^{\circ} \mathrm{C}$, on remarque que la survie des différentes bactéries testées est plus longue aux pH 6.2, 7.2, 8.4 (tableau 1). Les courbes des évolutions des différentes bactéries présentent les mêmes allures et se caractérisent par une décroissance lente de leur abondance. Les temps de survie d'E. coli et d'A. hydrophila sont presque identiques $\left(T_{90}=73 \mathrm{~h}\right)$ mais 
différents de celui de $S$. typhimurium $\left(T_{90}=142 \mathrm{~h}\right)$, bactérie qui survit toujours mieux que les autres bactéries dans les mêmes conditions expérimentales. A $\mathrm{pH} 9.6$ et à $12^{\circ} \mathrm{C}$ les courbes des évolutions des abondances indiquent une réduction du temps de survie des trois bactéries mais ces temps de survie sont cependant supérieurs à ceux obtenus à $23^{\circ} \mathrm{C}$ (tableau 1). Ainsi à pH 9.6 la présence de $S$. typhimurium n'est plus détectable dans le milieu réactionnel maintenu à $23^{\circ} \mathrm{C}$ au bout de 4 jours alors qu'à $12^{\circ} \mathrm{C}$ il faut attendre 5 jours pour atteindre le même résultat.

Pour une température d'incubation de $4^{\circ} \mathrm{C}$ et aux mêmes $\mathrm{pH}$, la survie bactérienne est très différente par rapport à ce qui est observé aux températures 12 et $23^{\circ} \mathrm{C}$. A $4^{\circ} \mathrm{C}$ et aux pH $6.2,7.2$ et 8.4 les courbes d'évolutions des abondances de $E$. coli et de $S$. typhimurium sont comparables et ne pré sentent pas de diminution (fig. 3). Par contre à pH 9.6, les abondances présentent une diminution notable mais cependant moins prononcée que celles observées aux températures de $12^{\circ} \mathrm{C}$ et surtout de $23^{\circ} \mathrm{C}$ (tableau 1).

Tableau 1 Temps de survie bactérienne $\left(T_{90}\right)$ dans l'eau de sortie du lagunage à différents pH et à différentes températures d'incubation.

Table 1 Survival time $\left(T_{90}\right)$ of bacteria in water from the outflow of the lagoon at different $\mathrm{pH}$ and at different temperature incubation.

\begin{tabular}{|lcccccccc|}
\hline & \multicolumn{4}{c}{$4^{\circ} \mathrm{C}$} & \multicolumn{2}{c}{$12^{\circ} \mathrm{C}$} & \multicolumn{2}{c|}{$23^{\circ} \mathrm{C}$} \\
& $\mathrm{pH}: 6,2$ & $\mathrm{pH}: 7,2$ & $\mathrm{pH}: 8,4$ & $\mathrm{pH}: 9,6$ & $\mathrm{pH}: 7,2$ & $\mathrm{pH}: 9,6$ & $\mathrm{pH}: 7,2$ & $\mathrm{pH}: 9,6$ \\
\hline E. coli & + & + & + & 28 & 73 & 23 & 53 & 20 \\
A. hydrophila & 84 & 86 & 80 & 34 & 73 & 20 & 53 & 20 \\
S. typhimurium & + & + & + & 42 & 142 & 33 & 100 & 24 \\
\hline
\end{tabular}

+ : les abondances bactériennes restent stables durant toute l'expérience, $\mathbf{T}_{90}$ non calculable.

A 12 et a $23^{\circ} \mathrm{C}$, les $T_{\infty}$ obtenus aux pH 6.2 et 8.4 sont presque identiques à ceux obtenus à pH 7.2.

+ : bacterial abundances remain stable in all experiments. $T_{\infty 0}$ not calculable.

At 12 and $23^{\circ} \mathrm{C}, T_{s 0}$ at pH 6.2 and 8.4 are almost the very same to those obtained at $p H 7.2$.

Les évolutions des abondances de $A$. hydrophila à $4^{\circ} \mathrm{C}$ et aux $\mathrm{pH}$ autres que 9.6, présentent une différence importante avec celles obtenues pour $E$. coli et $S$. typhimurium. A cette même température d'incubation il n'existe pratiquement pas de différence dans la survie d'A. hydrophila entre les pH 6.2, 7.2, et 8.4. Cependant la survie d'A., hydrophila à $4{ }^{\circ} \mathrm{C}$ et à pH $6.2,7.2$ et 8.4 , comparée à celle obtenue aux températures de 12 et $23^{\circ} \mathrm{C}$ est supérieure.

D'après cette première étude de l'effet du pH-température sur la survie bactérienne, on peut conclure qu'à faible température $\left(4^{\circ} \mathrm{C}\right)$, A. hydrophila subit une réduction du temps de survie beaucoup plus importante que celle observée chez $E$. coli et $S$. typhimurium. Pour des températures de 23 et $12{ }^{\circ} \mathrm{C}$, la sensibilité d'E. coli et de $A$. hydrophila est presque semblable. A ces mêmes températures, $S$. typhimurium survit mieux que ces deux bactéries. 

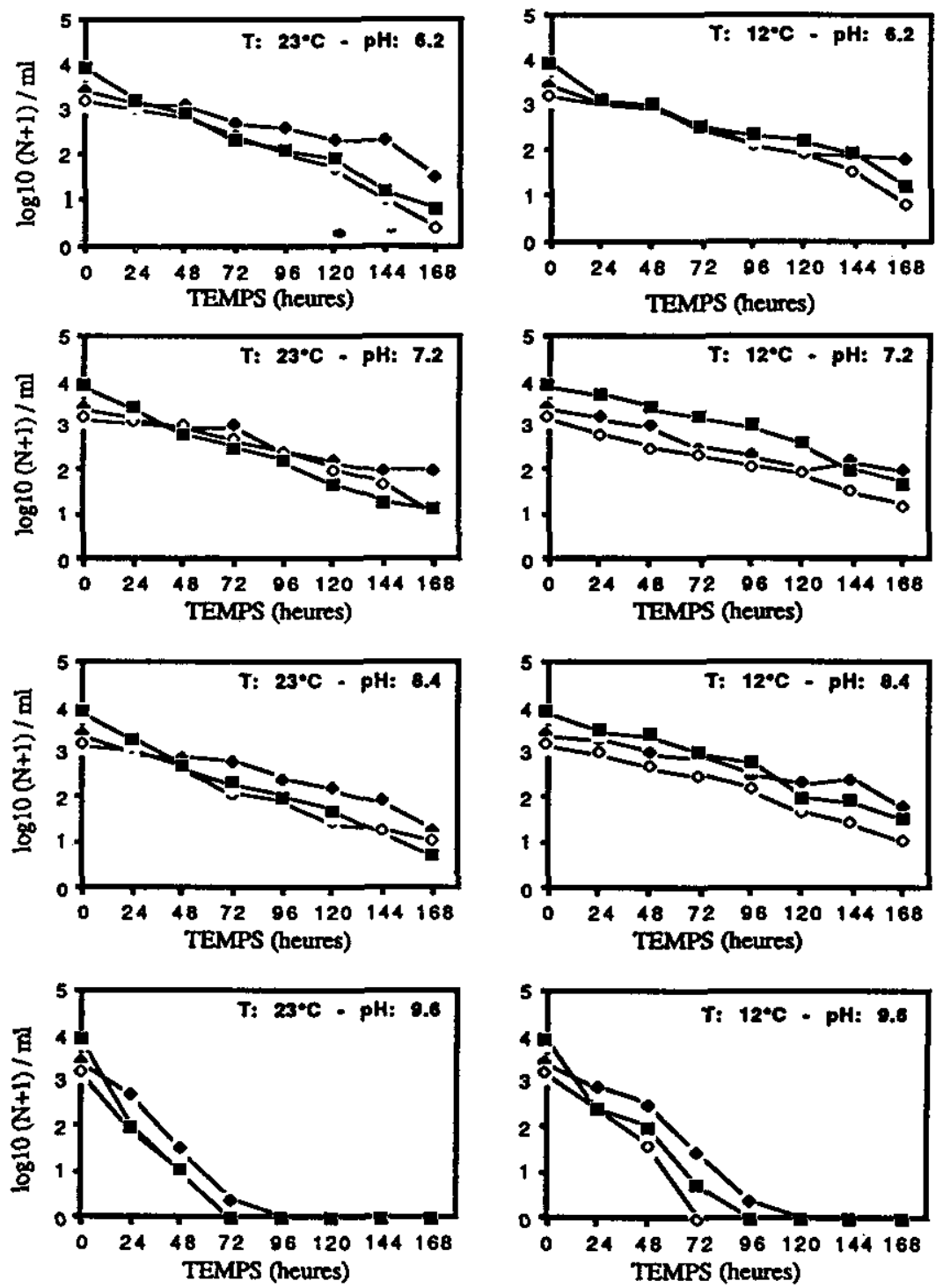

Figures 1 et 2 Evolution temporelle des abondances d'E. coli, d'A. hydrophila et de $S$. typhimurium dans l'eau de sortie du lagunage à différents $\mathrm{pH}$ et à différentes température.

Temporal evolution of E. coli, A. hydrophila and S.typhimurium in water from outflow of the aerobic lagoon at different $\mathrm{pH}$ and at different incubation temperature.

- : E. coli, $\diamond:$ A. hydrophila, $\bullet:$ S.typhimurium 

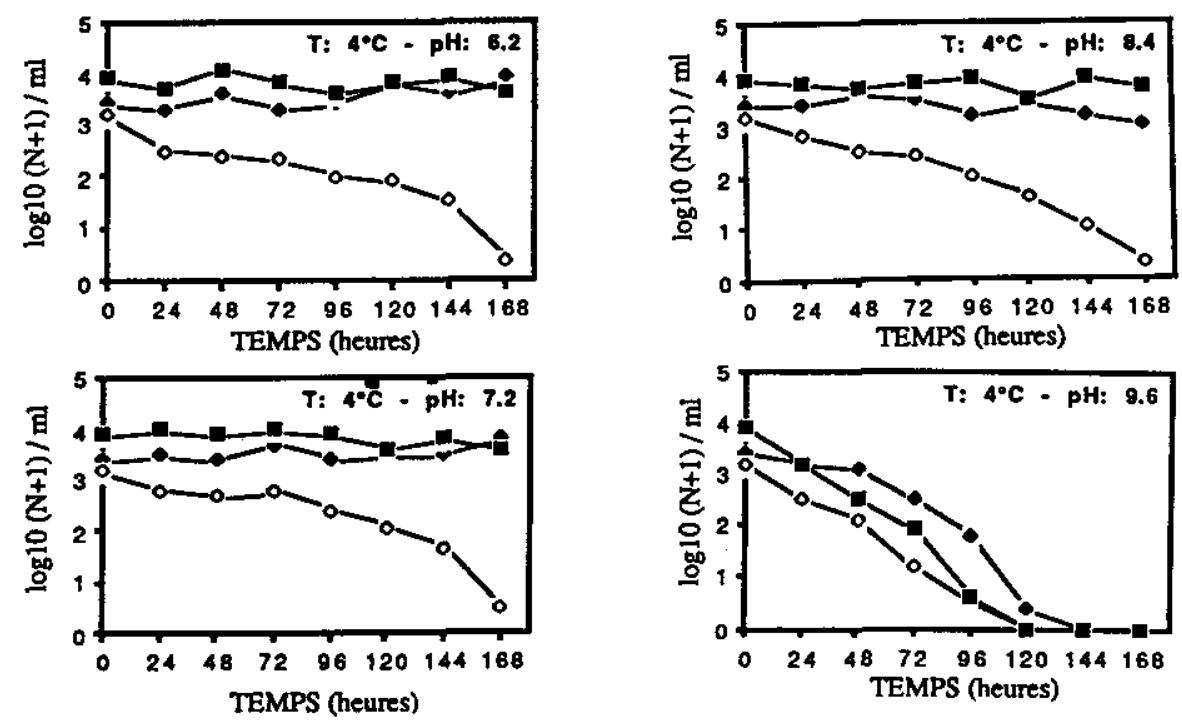

Figure 3 Evolution temporelle des abondances d'E. coli, d'A. hydrophila et de $S$. typhimurium dans l'eau de sortie du lagunage à différents $\mathrm{pH}$ et à une méme temperature $\left(4^{\circ} \mathrm{C}\right)$.

Temporal evolution of E. coli, A. hydrophila and S. typhimurium in water from outflow of the aerobic lagoon at different $\mathrm{pH}$ and at the same incubation temperature $\left(4^{\circ} \mathrm{C}\right)$.

- : E. coli, $\diamond:$ A. hydrophila, $\bullet:$ S.typhimurium

\section{pH-rayonnement solaire}

Les évolutions temporelles des abondances d'E. coli, de A. hydrophila et de $S$. typhimurium dans l'eau usée épurée par lagunage tamponnée à différents $\mathrm{pH}(7.2,8.4,9.6)$ et exposée au rayonnement solaire sont illustrées sur la figure 4. Dans l'échantillon à pH 7.2 protégé de toutes radiations solaires et considéré comme un témoin, E. coli, A. hydrophila et $S$. typhimurium présentent une évolution temporelle de leur abondance comparable (fig. 4A) Après 5 jours d'incubation à l'obscurité, les abondances d'E. coli, d'A. hydrophila et de $S$. typhimurium se trouvent réduites. Les $T_{80}$ respectifs sont de 87 , de 78 et de 87 heures (tableau 2). Dans le même milieu réactionnel ajusté au même pH 7.2 mais exposé aux rayonnement solaịre, l'évolution des abondances bactériennes des 3 espéces étudiées est différente de celle observée dans le témoin (fig. 4B). Les temps de survie $\left(T_{90}\right)$ relevés sont de 22 heures (E. coli), de 18 heures (A. hydrophila) et de 22 heures (S. typhimurium). En présence de radiations solaires, on assiste à une forte et rapide réduction de l'abondance bactérienne des 3 espèces étudiées (99\% après 48 heures d'exposition). Au-delà de 72 heures (fig. 4B), la présence des espèces bactériennes n'est plus détectable dans le milieu réactionnel (absence dans $10 \mathrm{ml}$ d'échantillon). En absence du rayonnement solaire et dans la même eau (non filtrée) épurée par lagunage et ajustée à pH 8.4, (fig. 4C) on observe un type d'évolution identique à celui rencontré dans l'échantillon témoin à pH 7.2. Quand ce 


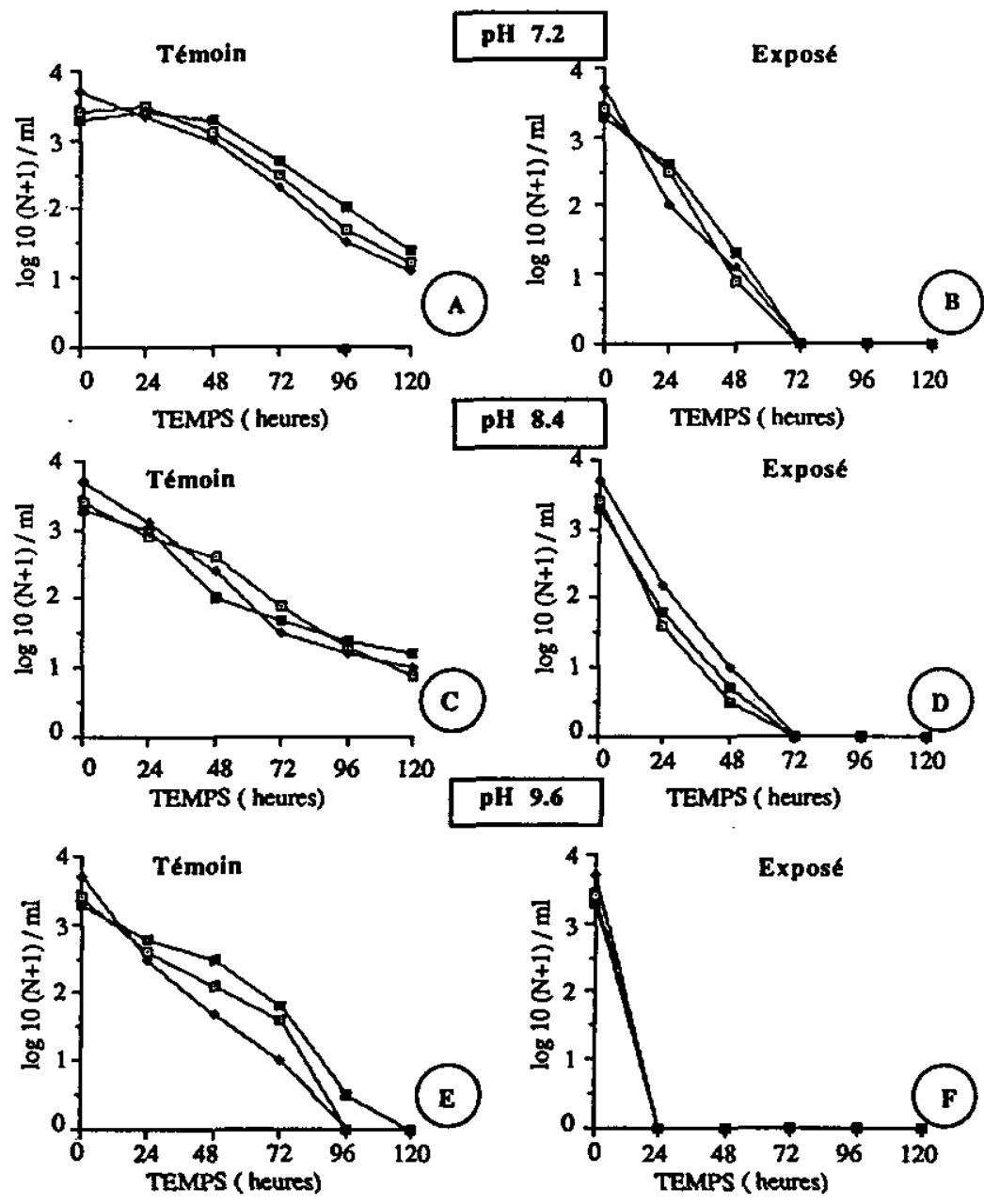

Figure 4 Evolution temporelle des abondances d'E. coli, d'A. hydrophila et de S. typhimurium dans l'eau de sortie du lagunage ajustée à différents $\mathrm{pH}$ et exposée au rayonnement solaire.

Temporal evolution of E. coli, A. hydrophila and S. typhimurium in water from the outflow of the aerobic lagoon adjusted at different $\mathrm{pH}$ and exposed to solar radiation.

$\neg \square:$ E. coli, $\rightarrow$ : A. hydrophila, $\rightarrow-:$ S.typhimurium

même milieu réactionnel (pH 8.4) est exposé au rayonnement solaire, l'abattement des abondances bactériennes est nettement supérieur à celui observé dans le témoin (fig. 4D). Pour une même quantité de radiations solaires (quantité moyenne/jour: 580 Joules $\mathrm{cm}^{-2}$ ) la diminution de l'abondance bactérienne atteint le même niveau mais plus rapidement que celle évaluée dans le milieu réactionnel à $\mathrm{pH} 7.2$. A pH 9.6 les trois bactéries étudiées présentent des survies plus courtes dans le milieu témoin (non exposé) que celles obser- 
vées dans les témoins pH 8.4 et pH 7.2 (tableau 2 et fig. 4E). La différence de survie d'E. coli d'A. hydrophila et de $S$. typhimurium à $\mathrm{pH} 9.6$ vis-à-vis de celles à pH 7.2 et 8.4 (témoins) est très significative au seuil de probabilité de $95 \%$. Exposées au rayonnement solaire (580 Joules $\mathrm{cm}^{-2} \mathrm{~J} \mathrm{Ju}^{-1}$ ) les trois souches bactériennes dans le même milieu réactionnel tamponné à pH 9.6 présentent des survies très raccourcies (tableau 2) non seulement par rapport à la non-exposition (fig. 4E) mais par rapport aux expositions avec des $\mathrm{pH}$ à 7.2 et 8.4. Cette survie n'excède pas 24 heures (absence d'u.f.c. dans $10 \mathrm{ml}$ d'échantilion). A noter que cette survie très raccourcie est identique pour $E$. coll et pour $S$. typhimurium $\left(T_{90}=6\right.$ heures) et légèrement inférieure pour A. hydrophila $\left(\mathrm{T}_{90}=4\right.$ heures). Ces résultats sont comparables à ceux obtenus par MARTIN et al. (1984) et BONNEFONT et al. (1990).

Tableau 2 Temps de survie bactérienne $\left(T_{90}\right)$ dans l'eau de sortie du lagunage exposée et non exposée au rayonnement solaire.

Table 2 Survival time $\left(T_{90}\right)$ of bacteria in water from the outflow of lagoon exposed and not exposed to solar radiation.

\begin{tabular}{|lcccccc|}
\hline & \multicolumn{2}{c}{$\mathrm{pH}: 7,2$} & \multicolumn{2}{c}{$\mathrm{pH}: 8,4$} & \multicolumn{2}{c|}{$\mathrm{pH}: 9,6$} \\
& non exposé & exposé & non exposé & exposé & non exposé & exposé \\
\hline E. coli & 87 & 22 & 80 & 20 & 24 & 6 \\
A. hydrophila & 78 & 18 & 75 & 16 & 22 & 4 \\
S. typhimurium & 87 & 22 & 80 & 20 & 35 & 6 \\
\hline
\end{tabular}

Dans ce genre d'étude il est possible de mettre en évidence une relation inverse entre le nombre de bactéries dénombrées (u.f.c.) et la quantité des radiations solaires (BELLAIR et al., 1977 et MC CAMBRIDGE et MC MEEKIN, 1981).

La décroissance du nombre de cellules bactériennes cultivables en fonction de la quantité de rayonnement solaire est-elle la conséquence d'un accroissement permanent de la mortalité ou bien ce phénomène est-il la résultante de phases successives de survie et de mortalité, consécutives à l'alternance naturelle des périodes nocturnes et diurnes ? Pour ce faire, il a été recherché quel était le comportement au cours de la période noctume des cellules d'E. coli ayant survécu à l'exposition au rayonnement solaire pendant la journée précédente.

Les résultats obtenus et illustrés sur la figure 5 indiquent que dans l'échantillon témoin (non exposé) les abondances bactériennes fluctuent moins que dans celui exposé au rayonnement solaire. Dans l'échantillon exposé et à l'issue des périodes noctumes on peut constater un accroissement des abondances d'E. coli par rapport à celles dénombrées à la fin de la période d'exposition précédente. Sous rayonnement solaire la survie d'une souche d'E. coli placée dans une eau usée épurée par lagunage stérilisée par filtration évolue par paliers successifs (phase de décroissance diurne, phase de croissance noctume) tant que les abondances ne sont pas inférieures à $1.10^{2}$ u.f.c. $\mathrm{mi}^{-1}$. Ces phases de croissances nocturnes pourraient être dues à des mécanismes de réparation de l'ADN endommagé (système SOS) pendant les phases diurnes d'exposition. Ces résultats confirment ceux de BELLAIR et al. (1977). 
En tenant compte de toutes les valeurs de dénombrement obtenues au cours de cette évolution jour/nuit et en traçant la droite de régression du $\log _{10}$ du nombre de bactéries en fonction du temps, on peut estimer un coefficient de mortalité $\left(K=0,043 \mathrm{~h}^{-1}\right)$ qui est la pente de la droite de régression et qui donne un temps de survie $\left(T_{90}\right)$ correspondant à 23 heures $\left(K=1 / T_{90}\right)$. L'équation obtenue est :

où :

$$
Y=-0,043 X+4,8\left(R^{2}=0,71\right)
$$

$Y=\log _{10}$ du nombre de bactéries

$X=$ temps (heures)

$\mathrm{R}^{2}=$ coefficient de détermination

En tenant compte des seules valeurs diurnes (14 H GMT) l'équation de régression devient :

$$
Y=-0,043 X+4,4 \quad\left(R^{2}=0,9\right)
$$

Puisque les deux équations présentent la même pente, un modèle peut être ajusté aux seules valeurs obtenues à 14 heures (heure GMT marqué par des flèches sur la figure 5).

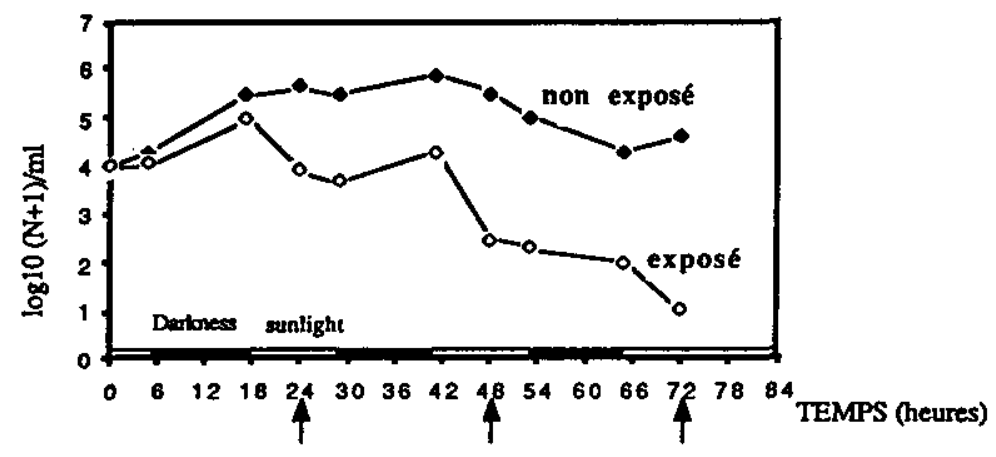

Figure 5 Evolution temporelle des abondances d'E. coli dans l'eau de sortie du lagunage (pH 8.3) exposée et non exposée au rayonnement solaire en fonction du rythme jour/nuit.

Temporal evolution of $E$. coli in water from the outflow of the aerobic lagoon ( $\mathrm{pH}$ 8.3) exposed and not exposed to solar radiation according to mythm day/night.

$\uparrow$ (dénombrement bactérien à $14 \mathrm{~h}$ GMT)

$\uparrow$ (Bacterial enumeration at 2 : 00 PM. GMT)

Les prédictions de ce type de modèle sont satisfaisantes pour des durées égales à 24 heures ou leur multiple. Les phases de décroissance ou de stabilité des périodes noctumes ne peuvent être prédites par ce modèle du fait de sa linéarité et de sa pente négative.

Quant au comportement d'une souche d'E. coli * sauvage * vis-à-vis du facteur rayonnement (fig. 6), le temps de survie de cette souche exposée au rayonnement solaire ( $T_{90}=26$ heures) et non-exposée $\left(T_{90}=125\right.$ heures) est légèrement supérieur à celui d'E. coli 0126 : B16 dans les mêmes condi tions ( $T_{90}=18$ heures, $T_{90}=111$ heures). La valeur du rapport calculé entre 
le $T_{90}$ (non exposé) et le $T_{90}$ (exposé) informe sur la sensibilité bactérienne au rayonnement solaire. Pour E. coli sauvage la valeur du rapport est de 5,95 et 6,16 pour $E$. coli O126: B16. Les deux souches bactériennes présentent donc une sensibilité comparable au rayonnement solaire dans une eau usée épurée par le lagunage après stérilisation par filtration.

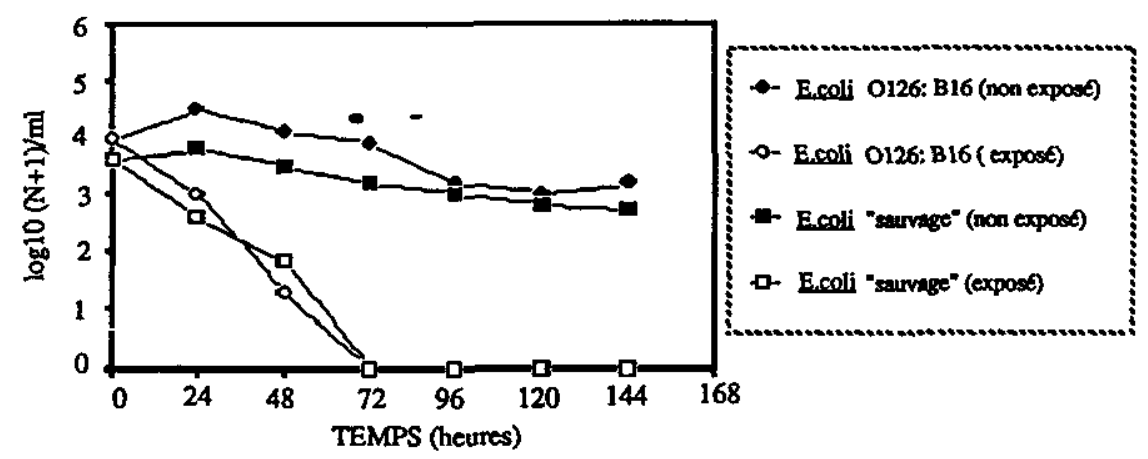

Figure 6 Evolution temporelle des abondances d'E. coli 0126: B16 et d'E. coli * sauvage * dans l'eau de sortie de lagunage exposée et non exposée au rayonnement solaire.

Temporal evolution of E. coli O126: B16 and E. coll isolated from natural ecosystem, in water from outflow of the aerobic lagoon exposed and not exposed to solar radiation.

\section{4 - DISCUSSION}

Les résultats obtenus au cours de cette étude montrent que les basses températures $\left(4^{\circ} \mathrm{C}\right)$ favorisent la survie d'E. colli et de $S$. typhimurium dans l'eau usée épurée par lagunage et non celle de $A$. hydrophila. Aux différentes températures testées, $S$. typhimurium survit mieux qu'E. coli et $A$. hydrophila. Cependant MC CAMBRIDGE et MC MEEKIN (1980) ont décrit que la survie d'E. coli et de $S$. typhimurium dans des eaux estuariennes à des températures différentes $(6.2 ; 10.3 ; 15.3 ; 20.6$ et 26.9$)$ est identique.

Le temps de survie des trois souches bactériennes testées n'est pratiquement pas influencé lorsque l'eau usée épurée par lagunage est à pH $6.2 ; 7.2$ et 8.4. Par contre le méme milieu à pH 9.6 entraîne une forte diminution du temps de survie des trois souches et ce principalement lorsque la température est élevée $\left(23^{\circ} \mathrm{C}\right)$. Les $\mathrm{pH}$ alcalins (9.6) étant bactéricides vis-à-vis d'E. coli, d'A. hydrophila et de $S$. typhimurium doivent agir et contrôler la dynamique des bactéries d'intérêt sanitaire dans les milieux aquatiques. PARHAD et RAO (1974) ont noté l'existence d'une corrélation entre l'augmentation du pH ( $\mathrm{pH}$ 9.2) et une diminution des abondances des coliformes dans une eau 
usée stérile. Cependant les pH alcalins aussi élevés (9.6) ne sont atteints que dans des milieux aquatiques le plus souvent artificiel (lagunage) où se développent des blooms phytoplanctoniques très importants. L'alcalinité du milieu se créait par suite de la consommation du $\mathrm{CO}_{2}$ par le phytoplancton. Ces développements phytoplanctoniques étant sous la dépendance entre autre de l'augmentation de la température de l'eau, ainsi la survie bactérienne dans ces écosystèmes aquatiques est avant tout sous la dépendance de la température.

L'action de la température sur la survie bactérienne dépend d'autres facteurs tels que les substances nutritives. Dans un milieu aquatique où les nutriments existent en concentrations élevées, la survie voire même la croissance bactérienne peuvent se produire si les températures sont proches de celle favorisant la croissance optimale de la bactérie. A l'inverse quand les substances nutritives font défaut, la survie est alors favorisée par les basses températures qui limitent les dépenses énergétiques de la cellule bactérienne par une diminution de son activité métabolique. Cependant dans l'eau de sortie du lagunage, bien que les substances nutritives soient présentes, une température de $23^{\circ} \mathrm{C}$ a conduit à une diminution des abondances bactériennes. II est possible d'émettre une hypothèse sur la cause de cette diminution. Une température de lordre de $23^{\circ} \mathrm{C}$ pourrait augmenter la constante de diffusion de produits toxiques présents dans le milieu réactionnel et favoriser ainsi leur contact avec la cellule bactérienne et par suite permettrait leur transport à l'intérieur de la cellule. Les températures élevées (de l'ordre de $25^{\circ} \mathrm{C}$ ) peuvent aussi agir indirectement sur la survie bactérienne par, soit la stimulation de la multiplication des prédateurs bactériens, soit en les rendant plus actifs sur le plan de leur prédation d'autant plus que ceux-ci se trouvent dans une gamme thermique de 20 à $25^{\circ} \mathrm{C}$ (ENZINGER et COOPER, 1976 ; MC CAMBRIDGE et MEEKIN, 1980). Les basses températures (de l'ordre de $4^{\circ} \mathrm{C}$ ) peuvent entraîner une meilleure survie par le fait que le métabolisme des bactéries mésophiles étant très ralenti, les produits toxiques présents en même temps que les fortes concentrations de nutriments ne sont que très lentement métabolisés (LESSARD et SIEBURTH, 1983). D'après les résultats obtenus on remarque que les températures basses limitent les effets des $\mathrm{pH}$ défavorables à la survie bactérienne : à pH 9.6 et à une température de $23^{\circ} \mathrm{C}$, le $\mathrm{T}_{90} \mathrm{~d}^{\prime} E$. coli est de 20 heures tandis qu'au même $\mathrm{pH}$ et sous une température d'incubation de $4^{\circ} \mathrm{C}$, le $\mathrm{T}_{90}$ est de $73 \mathrm{H}$. On peut supposer qu'à ces températures les échanges cellulaires se trouvent diminués et que, par conséquent, l'effet du $\mathrm{pH}$ se trouve limité. Ces résultats sont en accord avec ceux de CONNER et al. (1986). Cet auteur a montré que les basses températures $\left(5^{\circ} \mathrm{C}\right)$ peuvent assurer une protection des cellules de Listeria monocytogenes contre les $\mathrm{pH}$ acides ( $\mathrm{pH} 4,2)$.

Dans la littérature scientifique, il est fait mention que les basses températures permettent une prolongation de la survie bactérienne. Ainsi les résultats obtenus au cours de ce travail indiquent qu'au contraire la survie d'A. hydrophila se trouve réduite par des températures de l'ordre de $4^{\circ} \mathrm{C}$ si on la compare à celle de $E$. coli et de $S$. typhimurium à la même température. Ceci explique la dynamique cyclique de cette bactérie lors de l'épuration des eaux usées par lagunage à savoir faible abondance hivernale et forte abondance estivale (MONFORT, 1990). La réduction du temps de survie d'A. hydrophila par 
les basses températures pourrait être due à une augmentation de la mortalité par diminution de l'activité enzymatique. Elle pourrait être due aussi à l'incapacité des cellules bactériennes de se développer sur milieux de culture après un certain temps de contact avec des basses températures (bactérie * stressée *, non cultivable, ZASKE et al., 1980). Le temps de survie "cellulaire" pourrait être supérieur au temps de survie apprécié par la " colonie ". Cette survie serait donc sous estimée.

D'après les résultats obtenus le rayonnement solaire joue un rôle important dans l'abattement des coliformes fécaux et de certaines bactéries pathogènes lors de l'épuration par lagunage. II faut noter que l'action simultanée du $\mathrm{pH}$ et du rayonnement solaire est plus importante sur la survie bactérienne que si l'un des facteurs agit seul. $\mathrm{A}$ pH 9.6 et en présence du rayonnement solaire, le taux de réduction d'E. coli, de $A$. hydrophila et de $S$. typhimurium est plus grand que celui évalué au même $\mathrm{pH}$ mais à l'obscurité. Cette plus forte réduction bactérienne observée pourrait être expliquée par le fait que les souches bactériennes ayant subi dans un premier temps les effets nocifs des $\mathrm{pH}$ alcalins deviennent plus sensibles aux effets du rayonnement solaire. Les radiations solaires peuvent agir indirectement sur la survie bactérienne en stimulant l'activité prédatrice vis-à-vis des bactéries (MC CAMBRIDGE et MC MEEKIN, 1981). Cet effet indiscutable du facteur rayonnement solaire sur la survie bactérienne explique en partie les variations cycliques saisonnières des abondances des coliformes fécaux dans différents bassins de lagunage (BAHLAOUI, 1990). Le mode d'action du rayonnement sur les bactéries a été abordé dans plusieurs travaux (TUVESON, 1983 ; SAMMARTANO et TUVESON, 1985). Ces auteurs montrent que par la fraction UV des radiations solaires celles-ci ont un effet bactéricide. Ces radiations agissent sur la cellule en induisant des dommages par libération des ions peroxydes qui se forment par réduction du radical $\mathrm{O}^{2-}$ suite à une réduction incomplète de $\mathrm{O}_{2}$ durant la respiration bactérienne (GOERLICH, 1989).

Ces peroxydes agissent sur la cellule bactérienne en la rendant perméable aux sels inorganiques changeant de ce fait sa pression osmotique. Cette action bactéricide des UV surtout les UV.B (280-320 nm) sur la survie bactérienne dépend, entre autres, de la turbidité de l'eau (MEZRIOUl, 1987). En effet la matière organique en surface absorbe la majorité des UV et protège les cellules bactériennes contre les effets du rayonnement solaire. CHANG et al. (1985) ont montré que les petites particules inférieures à $10 \mu \mathrm{m}$ peuvent protéger la cellule bactérienne contre les effets des UV.

\section{CONCLUSION}

Dans les milieux aquatiques, la température est l'un des facteurs qui intervient dans les variations des abondances bactériennes. Les faibles températures de l'ordre de $4^{\circ} \mathrm{C}$ augmentent la survie bactérienne d'E. coli et de $S$. typhimurium et réduisent celle de $A$. hydrophila. A noter aussi que les 
faibles températures $\left(4^{\circ} \mathrm{C}\right)$ diminuent l'effet des $\mathrm{pH}$ alcalins sur la survie bactérienne. De plus il faut avoir à l'esprit que la température agit au moins de façon aussi importante sur les autres éléments biotiques d'un écosystème aquatique que sur les bactéries et qu'in situ ces effets indirects de la température sont à prendre en compte.

Parmi les autres facteurs contrôlant la dynamique bactérienne dans les milieux aquatiques, le rayonnement solaire joue un rôle non négligeable sur les évolutions des abondances d'E. coli, de $S$. typhimurium et d'A. hydrophila. Toutefois il faut noter que soes rayonnement solaire, cette réduction bactérienne est beaucoup plus accentuée lorsque le $\mathrm{pH}$ du milieu réactionnel est alcalin (pH 9.6). L'action simultanée du $\mathrm{pH}$ et du rayonnement solaire sur la survie bactérienne est plus importante que lorsque l'un des facteurs est pris seul. Pour une même température d'incubation les $\mathrm{pH}$ alcalins (pH 9.6) entraînent une réduction des abondances bactériennes plus importante que celle observée à pH 6.2, 7.2 ou 8.4.

Selon le rythme nycthéméral l'existence de phases successives de décroissance et de croissance bactérienne montre en fait que la diminution du nombre de cellules cultivables n'est pas un phénomène linéaire. Un modèle pouvant prédire les abondances bactériennes avec un pas de temps de 24 heures ou leur multiple a été établi. Sur la base d'un recueil d'un plus grand nombre de données il serait vraisemblablement possible d'ajuster de façon plus satisfaisante un modèle polynomial qui permettrait de prédire avec un pas de temps plus court les concentrations d'E. coli et d'autres bactéries d'intérêt sanitaire. De plus pour optimiser les effets du rayonnement solaire sur la réduction des abondances bactériennes, il serait envisageable d'effectuer les rejets encore contaminés en plein jour et dans des eaux les plus claires possibles. Ce facteur auto-épurateur est primordial dans le fonctionnement des lagunes dans les pays à climat aride où la quantité du rayonnement solaire est importante toute l'année.

\section{RÉFÉRENCES BIBLIOGRAPHIQUES}

BAHLAOUI A., 1990. Lagunage à hautrendement expérimental : dynamique de différents groupes bactériens et performances épuratrice sanitaire. Doctorat ofUniversité de Montpollier., $186 \mathrm{p}$.

BALEUX B., TROUSSELLIER M., 1983. Evolution des bactéries témoins de contamination fécale et de Pseudomonas aeruginosa et Aeromonas hydrophila dans un ourvage dépuration des eaux usées par lagunage (Mèze). Tech. Sci. Mun., 7, 361-370.
BELLAIR J.T., SMITH G.A.P. WALLIS I.G., 1977. Significance of diurnal variations in fecal coliform dieoff rates in the design of ocean outfalls. J. Water Pollut. Control Fed., 49, 2022-2030.

BONNEFONT J.L., MARTIN Y.V., GUIENNET B., 1990. Etude expérimentale de la décroissance des bactéries fécales en milieu marin : quantification, facteurs impliqués. Wat. Res., 24 (3), 267-273. 
CHAMBERLIN C.E., MITCHELL R., 1978. A decay model for enteric bacteria in natural waters, p. 325-348. In R. Mitchell (ed.). Water Pollution Microbiology, Vol. 2, John Wiley \& Sons, New York.

CHANG J.C.H., OSSOF C.S.F., LOBE D.C., DORFMAN M.H., DUMAIS C.M., QUALLS R.G., JOHNSON J.D., 1985. UV inactivation of pathogenic and indicator microorganisms. Appl. Environ. Microbiol., 49, 13611365.

CONNER D.E., BRACKETT R.E., BEUCHAT L.A., 1986. Effect of temperature, sodium chloride and $\mathrm{pH}$ on growth of Listeria monocytogenes in Cabbage Juice. Appl. Environ. Microbiol. 52, 59-63.

DAVEY K.R., 1989. A predictive model for combined temperature and water activity on microbial growth during the growth phase. Appt. Bacteriol., 67, 483-488.

ENZINGER R.M., COOPER R.C., 1976. Role of bacteria and protozoa in the removal of Escherichia coli from estuarine waters. Appl. Environ. Microbiol., 31, 758-763.

FRONTIER S., 1981. Méthodes statistiques: Application à la biologie, la médecine et l'écologie. Première édition, Masson, Paris, 246 p.

FUJIOKA R.S., HASHIMOTO H.H., SIWAK E.B. YOUNG R.H.F., 1981. Effect of sunlight on survival of indicator bacteria in seawater, Appl. Environ. Microl., 41, 690-696.

GOERLICH O., QUILLARDET P., HOFNUNG M., 1989. Induction of the SOS Response by hydrogen peroxide in various Escherichia coli mutants with altered protection against oxidative DNA damage. J. Bacteriol., 171 (11), 6141-6147.

GRAHAM J.J., SIEBURT Mc N J., 1973. survival of Salmonella typhimurium in artificial and coastal seawater. Rev. Inter. Oceanogr. Med, 29, 5-298.

GAMESON D.J., GOULD G., 1985. Bacterial mortality. In Investigation of sewage discharges to some British coastal waters, Part 2, chap. 8.

HICKEY E.W., HIRSHFIELD I.N., 1990. Low - pH - induced effects on patterns of protein synthesis and on internal $\mathrm{pH}$ in Escherichia coli and Salmonella typhimurium. Appl. Environ. Microbiol. 56 (4), 1038 1045.
LEGENDRE P., BALEUX B., TROUSSELLIER M., 1984. Dynamics of pollution-indicator and heterotrophic bacteria in sewage treatment lajgoon. Appl. Environ. Microbiol. 48, 586-593.

LESSARD E.J., SIEBUTH J.M., 1983. Survival of natural sewage populations of enteric bacteria in diffusion and bath chambers in the marine environment. Appl. Environ. Microbiol., 45, 950-959.

MARTIN G., LAIFA A., LAPLANCHE A., 1985. Approche du rōle de certaines substances dans la desinfection par rayonnement U.V. $38^{e}$ Journées Internationales du Cebedeau, Bruxelles Edition Cebedoc. Liege.

MARTIN Y.V., LELONG P., TANGUY B., MAGURNO C., BONNEFONT J.L., EQUEL J.C., 1984. Qualité des eaux littorales. Disparition des coliformes fécaux d'un effluent urbain en milieu marin - Etude expérimentale. Vie Mar. Ministère de l'Environne ment (1982) Rejet en mer. Guide pour I'Etude du Milieu Marin.

Mc CAMBRIDGE J. Mc MEEKIN T.A., 1980. Effect of temperature on activity of predators of Salmonella typhimurium and Escherichia coli in estuarine water. Aust. J. Mar. Freshwater Res., 31, 851-855.

MC CAMBRIDGE J. Mc MEEKIN T.A., 1981. Effect of solar radiation and predacious microorganisms on survival of fecal and other bacteria. Appl. Environ. Microbiol., 41, $1083-1087$.

Mc FETERS G.A. STUART D., 1972. Survival of coliform bacteria in naturel waters : field and laboratory studies with membrane-filter chambers. Appl. Microbiol. 24, 805-811.

MEZRIOUI N, 1987. Etude expérimentale des effets du $\mathrm{pH}$, du rayonnement solaite et de la temperature sur la disparition des bactéries d'intérêt sanitaite et évaluation de la résistance aux antibiotiques d' $E$. coll brs de l'épuration des eaux usees. These de Doctorat d"Universite de Montpellier, 186 p.

MITCHELL D.O., STARZYK M.J., 1975. Survival of Salmonella and other indicator microorganisms. J. Canad. Microbiol., 21, 1420-1421.

MOELLER I.R., CALKINS J., 1980. Bactericidal agents in wastewater lagoons and lagoon design. J. Water. Poll. Control Fed, 52, 2441- 2451. 
MONFORT P., BALEUX B., 1990. Dynamics of Aeromonas hydrophila, Aeromonas caviae, and Aeromonas sobria in a sewage treatment pond. Appl. Environ. Microbiol. 56, 1999-2006.

PARHAD N.M. RAO N.U., 1974. Effects of pH on survival of Escherichia colf. J. Water Poll. Control Fed. 48, 980-986.

ROGOL M., SECHTER I., GRINBERG L., GERICHTER C.B., 1979. PribXyleseAmpicilline-Agar, a new selective medium for the isolation of Aeromonas hydrophila. J. Med. Microbiol., 12, 229-231.

ROUF M.A., RIGNEY M.M., 1971. Growth temperatures and temperatures characteristics of Aeromonas. Appl. Environ. Microbiol., 22, 503-506.

SAMMARTANO L.J. TUVESON R.W., 1985. Hydrogen peroxide induced resistance to broad-spectrum near-ultraviolet light $(300-$
$400 \mathrm{~nm}$ ) inactivation in Escherichia coli. Photochem. Photobiol., 41, 367-367.

TROUSSELLIER M., LEGENDRE P. BALEUX B., 1986. Modeling of the evolution of bacterial densities in an eutrophic ecosystem (sewage lagoon). Microbial Ecol., 12, 355379.

TUVESON R.W., PEAK J.G., PEAK M.J., 1983. Single-strand DNA breaks induced by $365 \mathrm{~nm}$ radiation in Escherichia coli strains differing in sensitivity to near and far UN. Photochem. Photobiol., 37, 109-112.

VASCONCELOS G.J., SWARTZ R.G., 1976. Survival of bacteria in seawater using a diffusion chamber apparatus in situ. Appl. Environ. Microbiol., 31, 913-920.

ZASK S.K., DOCKINS W.S., Mc FETERS G.A., 1980. Cell envelope damage in Escherichia coll caused by short-term stress water. Appl. Environ. Microbiol., 40, 386-390. 